MMP-9) revealed changes in protein expression between asphyxia and control cases.

Conclusion Placental biopsies collected at up to 90 minutes after delivery show stable gene and protein expression and may provide useful early biomarkers in NE.

\section{CEREBELLAR AND THALAMIC GROWTH IN PRETERM INFANTS IN RELATION TO BIRTH WEIGHT (BW)}

doi:10.1136/archdischild-2012-302724.1063

'E Andrew, ${ }^{1} \mathrm{~J} \mathrm{Siu},{ }^{2} \mathrm{VJ}$ Pelling, ${ }^{3} \mathrm{H}$ Rabe, ${ }^{2} \mathrm{P}$ Amess, ${ }^{2} \mathrm{JR}$ Fernandez Alvarez. ${ }^{1}$ Brighton and Sussex Medical School; ${ }^{2}$ Brighton and Sussex University Hospitals NHS Trust; ${ }^{3}$ Brighton and Sussex University Hospitals, Brighton and Sussex Medical School, Brighton, UK

Background Reduced cerebellar/thalamic growth affects neurodevelopment. The exact mechanisms are unknown.

Aims To compare cerebellar/thalamic growth of preterms in relation to BW and weight at 36 weeks' corrected (W36).

Methods Retrospective matched cohort-analysis: 4 BW groups matched for maternal smoking, chorioamnionitis, antenatal steroids, delivery mode, multiples and gender.

Exclusions growth restriction, congenital anomalies.

Study Variables (table1) gestational age (GA), BW, W36, head circumference at birth/36 weeks (HC/HC36), transverse cerebellar/ thalamic diameter at birth/36 weeks (TCD/TTD and TCD36/ TTD36).

Confounding variables ventilation days $(\mathrm{V})$, oxygen requirement 36 weeks' corrected $\left(\mathrm{O}_{2}\right)$, postnatal steroids (PS), NEC, days antibiotic treatment $(\mathrm{ABX})$, days parenteral nutrition (TPN), phototherapy, IVH, PVL.

Statistics median (quartiles, $25^{\text {th }} / 75^{\text {th }}$ ), Friedman-/Cochran-Test.

There was a statistically significant difference in $\mathrm{V}, \mathrm{PS}, \mathrm{O}_{2}, \mathrm{ABX}$, TPN, but not NEC, phototherapy, IVH, PVL.

Conclusion Cerebellar growth is more resilient than thalamus or cerebrum to the negative effects of established risk factors for poor neurodevelopmental outcome.

\section{NEUROLOGICAL INVOLVEMENT OF ENTEROVIRUS INFECTION IN YOUNG INFANTS WITH SEPSIS-LIKE ILLNESS}

doi:10.1136/archdischild-2012-302724.1064

${ }^{1}$ EP De Jong, ${ }^{2}$ EPM Van Elzakker, ${ }^{3} \mathrm{H}$ Holscher, ${ }^{4} F J$ Walther, ${ }^{1} \mathrm{~F}$ Brus. ${ }^{1} \mathrm{Juliana}$ Children's Hospital; ' 2 Medical Microbiology; ${ }^{3}$ Radiology, HAGA Hospital, The Hague; ${ }^{4}$ Paediatrics, Division of Neonatology, Leiden University Medical Center, Leiden, The Netherlands

Introduction Enterovirus (EV) infection is common in young infants. Amongst those requiring intensive care seizures and cerebral white matter abnormalities with serious neurologic sequelae have been reported. We questioned whether similar neurologic features occur in less seriously ill infants with EV infection.
Methods From august 2011 onward we included children under 90 days of age, admitted to a medium care unit with sepsis-like illness due to $\mathrm{EV}$ infection but not requiring intensive care. Cerebral ultrasound imaging was performed during hospital stay, cerebral MRI and hearing screening 4-6 weeks post-infection. During all visits neurological examination was performed and developmental milestones determined.

Results Preliminary results of the first 13 infants included are presented. Seven had positive EV PCR in CSF and serum, 6 tested positive in serum only. None showed seizures or abnormal neurological examination at admission. Cerebral ultrasound was performed in $12 / 13$ infants and showed no abnormalities. Cerebral MRI was performed in 10/13 infants; one showed diffuse white matter abnormalities in the frontal and occipital lobe. Hearing screening was normal in all infants. Neurological examination 4-6 weeks after infection showed slight hypertonia of the lower extremities in one infant. At 6 months of age all infants had normal neurologic examination.

Conclusion At 4-6 weeks following EV infection cerebral white matter abnormalities were found on MRI in one infant, whereas a slight hypertonia of the legs was found in another. At 6 months of age neurological examinations were normal in all infants. The long term implications of our findings are unclear.

\section{REFERENCE VALUES OF ULTRASOUND BRAIN MEASUREMENTS IN PRETERM NEWBORN INFANTS}

doi:10.1136/archdischild-2012-302724.1065

'JA Wroblewska, ${ }^{2} \mathrm{~J}$ Surmik, "I Maruniak-Chudek, ${ }^{3} \mathrm{~J}$ Swietlinski. 'Neonatal Intensive and Special Care, Silesian Medical University; ${ }^{2}$ Neonatal Intensive and Special Care, Upper Silesian Centre for Child Health, Katowice; ${ }^{3}$ Neonatal Intensive Care Unit, City Hospital, Ruda Slaska, Poland

Background and Aims Ultrasonography is noninvasive and commonly used for neuroimaging in premature newborns. Studies on brain measurement techniques and reference values of brain diameters are lacking. This paper aimed at

1. determining average diameters of cerebral ventricles, brain and cerebellum in preterm infants;

2. describing discrepancies between left and right sides, and between type of transducers used;

3. assessing possible relationships between diameters measured and gestational age, head circumference, birth length, birth weight, and gender.

Methods 132 newborn infants were enrolled and each subject had 34 diameters measured and 6 ratios calculated. Area of cavum septum pellucidum (ACSP), ventricular index (VI), ventricular width (VW), brain width (BW), third ventricle width (3VW), frontal horn width (FHW) were assessed in coronal plane. Evans (ER) and Johnson (JR) ratios were calculated. Midbody of lateral ventricle (MLV) and thalamo-occipital distance (TOD) were studied in parasagittal

Abstract 1063 Table 1

\begin{tabular}{lccccc}
\hline & $\mathbf{1 0 0 0 g}(\mathbf{N}=\mathbf{1 4})$ & $\mathbf{1 0 0 0} \mathbf{- 1 4 9 9 g}(\mathbf{N}=\mathbf{1 7})$ & $\mathbf{1 5 0 0 - 2 4 9 9 g}(\mathbf{N}=\mathbf{8})$ & $\mathbf{2} \mathbf{2 5 0 0} \mathbf{g}(\mathbf{N}=\mathbf{4 6})$ & $\mathbf{p}$-value \\
\hline GA(weeks) & $26(25-27)$ & $30(28-31)$ & $31(31-32)$ & $39(38-40)$ & $<0,001$ \\
BW(g) & $762(585-882)$ & $1200(1130-1390)$ & $1670(1573-1765)$ & $3345(2814-3668)$ & $<0,001$ \\
HC(cm) & $23.5(22.0-24,5)$ & $27(26.0-27,3)$ & $29.2(28.3-30,1)$ & $34.2(33.5-35,5)$ & $<0,001$ \\
TCD(cm) & $2.4(2.3-2.7)$ & $3.2(2.7-3.4)$ & $2.9(2.6-3.5)$ & $4.0(3.6-4.3)$ & 0.002 \\
TTD(cm) & $1.8(1.7-2.0)$ & $2.2(2.0-2,3)$ & $2.4(2.3-2,4)$ & $2.8(2.6-2,9)$ & $<0,001$ \\
W36(g) & $1702(1528-1850)$ & $2050(1800-2200)$ & $2250(2018-2350)$ & Not applicable & $<0,001$ \\
HC36(cm) & $29.8(28.4-31,6)$ & $32(31.0-32,6)$ & $32.3(30.3-32,8)$ & Not applicable & 0,001 \\
TCD36(cm) & $3.3(3.2-3.7)$ & $3.6(3.1-4.3)$ & $4.0(3.5-4.4)$ & Not applicable & 0.072 \\
TTD36(cm) & $2.4(2.3-2,6)$ & $2.4(2.3-2,5)$ & $2.7(2.6-2,9)$ & Not applicable & 0,028 \\
\hline
\end{tabular}

